fournal of Medical Genetics (1970). 7, 285.

\title{
Fluorescence Angiography and Inherited Degeneration of the Fundus Oculi
}

\author{
D. W. HILL*
}

Within the discipline of ophthalmology, fluorescence angiography has achieved popularity in the past decade as a method of investigation, demonstrating abnormalities in the retinal and choroidal circulations. Alterations in Bruch's membrane and the overlying pigment epithelium are seen as modifications in the pattern of underlying choroidal fluorescence. A large volume of literature has accumulated around the technique and its interpretation in various aspects of ocular disease. In this issue of the fournal of Medical Genetics dedicated to Professor Arnold Sorsby, it is appropriate to present a brief essay on the application of this technique to a subject of interest to him, genetically determined degenerations of the fundus oculi.

\section{Introduction}

A brief description of the method and appearances seen in the normal fundus must be given to orientate those not familiar with the technique. A modified retinal camera, having exciting and barrier filters in the illuminating and photographic light paths respectively, is used to photograph the fluorescent image of the fundus oculi to the exclusion of other detail. The dye is administered by rapid intravenous injection into an antecubital vein, and photography started a few seconds after the injection, to show in successive frames the passage of dye through the circulations of the fundus. It is seen first in the ciliary circulation as a sudden fluorescent blush over the whole fundus, mottled in form, derived from the choroid, which is seen through the overlying retinal pigment epithelium; this rapidly intensifies while dye enters the retinal arteries. In successive frames the dye is seen to enter the retinal veins, at first near the macula, then more peripherally; the capillary network is often visible while venous filling is developing, especially around the fovea, where increased density of retinal

\footnotetext{
* Address: Research Department of Ophthalmology, Royal College of Surgeons of England, Lincoln's Inn Fields, London W.C.2.
}

pigment epithelium obscures the choroidal fluorescence. In the normal fundus few details of the choroidal circulation are seen, but the mottled fluorescence already mentioned at first increases and later fades as the venous phase of retinal fluorescence proceeds. Fluorescein does not leak from healthy retinal vessels, in contradistinction to most other circulations including the choroidal, which are freely permeable. The dye in the tissue spaces of the choroid is prevented from passing into the retina by Bruch's membrane. One of the most striking features of the normal transit of dye through the retinal vessels is the formation of streamlines in the main veins (Fig. 1) due to the fact that circulation is more rapid in the macular region (about 2-3 seconds) than in the periphery (510 seconds), so that the return of dye is staggered. Apart from this inequality, regional differences of filling in the retina and choroid may be observed; in the latter case a dark area is seen which fills later than the surrounding areas. These regional differences may be constant, or transient, disappearing in a subsequent study.

\section{Generalized Fundus Dystrophy}

Generalized fundus dystrophy, described by Sorsby and often known eponymously, is characterized by dominant inheritance and onset, with exudative signs at the posterior pole of the eye, during the late fourth or early fifth decades of life. Central vision is lost and the final appearance is of widespread choroido-retinal atrophy, with irregular pigmentary clumping and choroidal sclerosis. Reports of the fluorescein appearances are sparse, but 12 members of one family in which there were 3 established cases were examined by Rosen and Leighton (1968). The angiogram of a patient with the late stage of the disease, examined at the Royal Eye Hospital, is shown in Fig. 2. The principal findings in the established stage of the disease are of irregularity in the background fluorescence from the choroid, with scattered areas which show increasing fluorescence after the completion of transit 


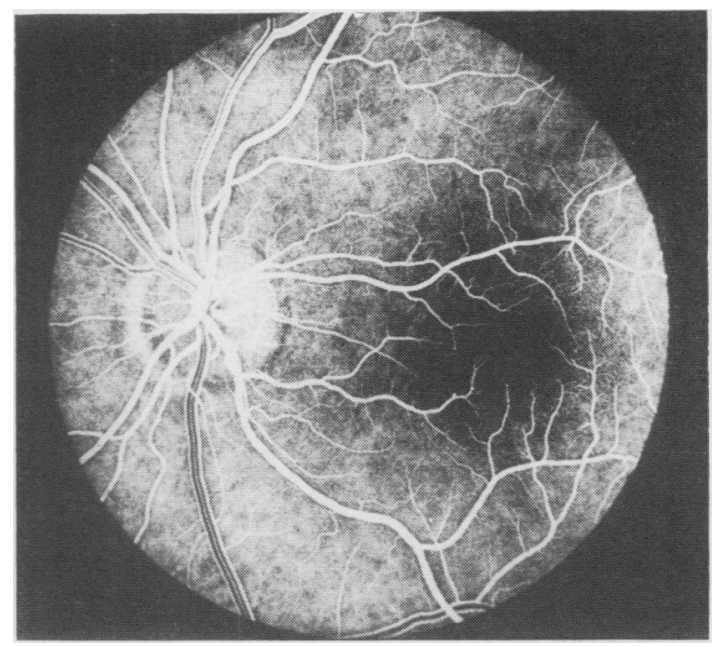

FIG. 1. The early venous phase of a fluorescence angiogram of the normal fundus in a young adult. The dye has a white image and outlines the retinal arterioles vividly. Some capillary details are seen around the macula (to the right) and the mottled choroidal fluorescence is reduced in this area. The main veins show streamlines of dye returning from the macula and adjoining areas interspersed with unstained (dark) peripheral blood. Fluorescence is also seen on and around the optic disc (to the left) from which the vessels radiate.

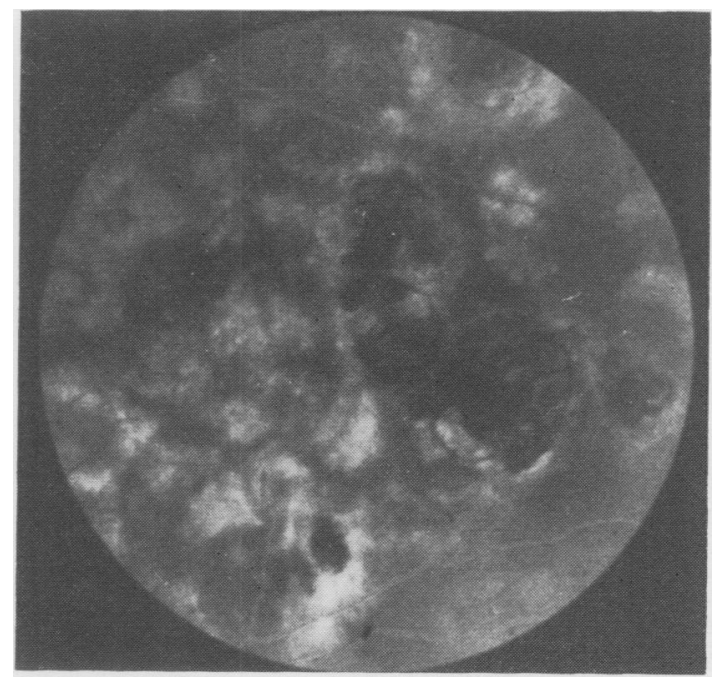

FIG. 2. Late phase of a fluorescence angiogram of a patient with generalized fundus dystrophy, centred on the macula region. Dye has almost passed from the retinal vessels, and a residual pattern of disturbed choroidal fluorescence is seen. A coarse pattern of abnormally intense fluorescence is interspersed with dark areas due to dense aggregations of retinal pigment, which appear as black patches on ophthalmoscopy.

of dye, but retain their original outline. These changes are ascribed to disturbances of the retinal pigment epithelium which allow more of the underlying choroidal fluorescence to be seen where the epithelium has become thin or absent, and hold back the fluorescence where it has aggregated. Angiograms of the exudative phase have not yet been reported, but one might expect to see a similar appearance to that found in the early stages of disciform degeneration, a number of foci of intense fluorescence from which the dye leaks slowly into the surrounding tissues, diffusing into the retina. Since blood is opaque to the fluorescent light, any haemorrhages present in the fundus would hide the background choroidal fluorescence by a shadow of uniform density.

\section{Retinitis Pigmentosa and Allied Diseases}

Retinitis pigmentosa in various forms is more commonly encountered than generalized fundus dystrophy; its clinical manifestations and variations are well known to ophthalmologists. Essentially a gradual degeneration and atrophy of the retinal receptor elements, starting in the equatorial zone of the fundus, it is accompanied by a fine pigment mottling of the fundus with aggregates of exuberant pigment around the retinal vessels. A variable degree of choroidal sclerosis and atrophy accompanies these changes. The fluorescence angio grams again show disturbance of the retinal pigmento epithelium, though in this instance it is situated in the equatorial region initially. Atrophy of the underlying pigment epithelium discloses the choroidal circulation, and where secondary sclerosis has occurred dye is seen in the choroidal arteries in the early phase of the angiogram, while later on the large vessels are silhouetted against a fluorescent background (Hill et al., 1964).

Choroideraemia shows in the fluorescence angiogram, retinal pigment epithelial changes, exposure of the large choroidal vessels, and finally complete loss of fluorescence in the late stages of atrophy. The macula remains intact until a late stage of the disease (Krill, Newell, and Chishti, 1968).

Accompanying all of these generalized atrophic disorders of the fundus is a gradual atrophy of the retinal circulation, shown clinically by attenuated vessels, and angiographically by slowing of the circulation, narrowing of the vessels, and loss of capillary detail.

Those fundus dystrophies in which colloid or other deep deposits in the retina accumulate show a pattern of fluorescence angiography which depends upon the extent of disturbance of the underlying retinal pigment epithelium in relation to the deposits. Where the pigment epithelium is disrupted by the deposit, a bright point of fluorescence, derived from the underlying choroid, is seen. Because of the 
persistence of fluorescence, some authors have suggested that the dye is taken up by the colloid material (Rubinstein and Paton, 1966), but part at least of this appearance is due to the persistence of fluorescein in the tissue fluid of the choroid. Not all colloid bodies fluoresce, and the outlines of the fluorescent image do not necessarily correspond exactly with the clinically visible deposit. A similar pattern is seen in fundus flavimaculatus (Ernest and Krill, 1966), though the correspondence between the clinical 'fishtail' deposits in the retina and the pattern of fluorescence is less obvious, and there is very little correspondence between the outline of the deposit and that of its fluorescent image (Brown and Hill, 1968).

\section{Macular Degeneration}

Passing from the generalized to the limited degenerations affecting the macula, the pattern of the fluorescence angiogram is built round the two reactions already described in connexion with generalized fundus dystrophy, retinal pigment epithelial disturbance, and exudative reaction with leakage of fluorescein. In some cases considerable pigmentary change is visible at the macula, with loss of vision but little or no angiographic abnormality, while in others angiography reveals widespread changes in excess of the clinical signs. An interesting example of fluorescence angiography in macular degeneration occurs in vitelliform degeneration (Best's disease), in which a pale cystic lesion is seen at the macula, graphically described as a 'honeydrop'. This lesion is compatible with good vision until the second or third decade of life when the cyst breaks up to be replaced by atrophy and pigmentation, and the central vision fails rapidly; only then does the fluorescence angiogram show any abnormality, as changes of retinal pigment epithelial disturbance, sometimes with a little leakage of dye, develop. The instances so far described do not of course cover the whole range of inherited fundus degeneration, which would not be appropriate to the present purpose; but it is hoped that sufficient has been said to indicate the type of changes to be expected, and further information can be obtained from the atlases mentioned in the select bibliography.

\section{Conclusion}

In drawing this essay to a close it is pertinent to inquire how fluorescence angiography can help in the diagnosis and management of inherited fundus disease. It will already have become apparent that the range of patterns revealed in degenerative lesions of the fundus is limited, though their distribution is variable. Further, it is clear that fluorescence angiography does not always show abnormalities in the presence of clinical signs and visual deterioration; and that on the whole electrophysiological tests are a more sensitive index of incipient disease (Geltzer and Berson, 1969), provided it is generalized.

The contribution of fluorescence angiography lies in providing a fresh mode of examination, giving information about the behaviour of the retinal pigment epithelium and Bruch's membrane. It may sometimes help to establish diagnosis by exposing abnormalities in the second eye, before clinical changes can be detected; but its real value lies in the fuller investigation of established cases. Recently the introduction of Xenon arc photocoagulation and laser coagulation has given fresh impetus to the study of these conditions, by providing a precise tool for the treatment of lesions of the posterior pole of the eye. In this connexion fluorescence angiography is indispensable, providing information unobtainable by any other means about the site of leakage in exudative reactions, which enables treatment to be directed to the correct site in an attempt to control the exudative reaction by sealing the leak. Such treatment can only be palliative, but it may delay the onset of severe visual loss for some time.

Professor Sorsby has contributed outstandlingly, in person and through the colleagues in his research department, to our knowledge of genetically determined fundus disease, its clinical manifestations and its biochemistry. This short essay is offered to show how a new technique may be employed to carry our knowledge of these diseases a stage further.

Thanks are due to Mr. J. D. Griffiths (research assistant) and Mr. S. Young (research photographer) of my department who prepared the fluorescence angiograms.

\section{REFERENCES}

Brown, N., and Hill, D. W. (1968). Fundus flavimaculatus. Two familial cases with macular degeneration. British fournal of Ophthalmology, 52, 849-852.

Ernest, J. T., and Krill, A. E. (1966). Fluorescein studies in fundus flavimaculatus and drusen. American fournal of Ophthalmology,
62, 1-6.

Geltzer, A. I., and Berson, E. L. (1969). Fluorescein angiography of hereditary retinal degenerations. Archives of Ophthalmology, 81, 776-782.

Hill, D. W., Dollery, C. T., Hodge, J. V., and Scott, D. J. (1964). Fluorescein studies of the choroidal circulation. Proceedings of the Royal Society of Medicine, 57, 500-502.

Krill, A. E., Newell, F. W., and Chishti, M. I. (1968). Fluorescein studies in diseases affecting the retinal pigment epithelium. American fournal of Ophthalmology, 66, 470-484.

Rosen, E. S., and Leighton, D. (1968). Fluorescein photography of generalized dominant fundus dystrophy. British fournal of Ophthalmology, 52, 828-832.

Rubinstein, K., and Paton, A. (1966). Fluorescein studies of macular changes. Transactions of the Ophthalmological Society of the United Kingdom, 86, 139-149. 


\section{SELECT BibLIOGRAPHY}

Hill, D. W. (1969). The techniques of fluorescence retinography. In The William Mackenzie Centenary Symposium on the Ocular Circulation in Health and Disease, p. 53. Ed. by J. S. Cant. Henry Kimpton, London.

Rosen, E. S. (1969). Fluorescence Photography of the Eye. A
Manual of Dynamic Clinical Ocular Fundus Pathology. Butterworths, London.

Shikano, S., and Shimizu, K. (1968). Atlas of Fluorescence Fundus Angiography. Igaku Shoin, Tokyo.

Wessing, A. (1968). Fluoreszenzangiographie der Retina; Lehrbuch und Atlas. Georg Thiene, Stuttgart. 